\title{
Knowledge of Malaysian university students towards swine-origin influenza A (H1N1) virus: A qualitative study
}

\section{Abstract}

Introduction: The study was aimed to explore the knowledge towards swine flu among university students.

Methods: An in-depth interview was conducted among 40 Students chosen randomly and asked to participate. The interviewers followed a standardized protocol to ensure that all the participants' interviews were conducted in a similar manner and that an identical set of questions were discussed. Data was analyzed manually.

Results: The majority of the participants were aware that H1N1 is a disease caused by Influenza A virus subtype H1N1. In terms of transmission, the majority of participants believed that the H1N1 might be transmitted from an infected person to a susceptible person; 12 out of 40 students thought that people might be infected by eating pork meat. Few students reported that the pig farmers and those in close contact with pigs were at high risk of the infection. Regarding preventions, half of the participants reported that H1N1 infection might be prevented by wearing face-masks, washing hands and avoiding close contact with infected patients.

Conclusion: Despite majority of the participants had the basic knowledge regarding $\mathrm{H} 1 \mathrm{~N} 1$ and its preventions measures such as wearing face-masks, washing hands and avoiding close contact with infected patients, some of them had some misconceptions about the infection, such as the infection might be transmitted by eating pork meat.

Keywords: Knowledge, H1N1, University Students, Qualitative Study.

\section{Redhwan Ahmed}

Al-Naggar ${ }^{1}$, Al-Jashamy Karim², Yuri V. Bobryshev ${ }^{3}$

1 Population Health and Preventive Medicine Department, Faculty of Medicine, Universiti Teknologi MARA, Malaysia, 47000.

2 Faculty of Medicine, SEGi University, Malaysia.

3 Faculty of Medicine, University of New South Wales, Sydney, NSW 2052, Australia.

\section{Contact information:}

Redhwan Ahmed Al-Naggar.

Faculty of Medicine, UiTM, Malaysia

”radhwan888@yahoo.com 


\section{Introduction}

A new strain of influenza A virus was announced by the World Health Organization (WHO) on late April 2009. The virus has been named after the animal "swine" which is considered to have begun the spread of the virus in human beings. It has proved to be a contagious virus among human beings. [1] It has spread in 74 countries within a very short period of time-from late April 2009 to 11 June 2009as nearly 30,000 were confirmed having caught it. In countries where good surveillance and testing procedures are available, the number of cases discovered is great; exceptions are few in such places. The spread of the virus has got out of systematic trace as it has spread in several countries. Therefore, further spread is likely inevitable. This could be the reason that made the $\mathrm{WHO}$ announce the $\mathrm{H} 1 \mathrm{~N} 1$ to be of phase 5 when it emerged. [2] Later the WHO re-announced it to be of phase 6 indicating the raise of influenza pandemic level. The influenza pandemic is now worldwide. [3].

Malaysia is no different from the rest of the world in this regard. In it, the first case announced to have had the H1N1 was on 16 May 2009. This announcement was made three days after a 21-year-old male student flying from the United States had arrived to Malaysia. [4] In almost a month and a few days later (7 July 2009), the Malaysian Ministry of Health reported the existence of 468 cases of H1N1 until that date. [5] In the light of all this and because of the fear and anxiety generated by the spread of the virus as well as the scarcity of H1N1 awareness documentations, this study was conducted to explore the awareness of swine flu among university students

\section{Methods}

An in-depth interview was conducted in May 2009 among 40 Students. Twenty of them from Bachelor of Medical Sciences (BMS) and the other 20 from Bachelor of Biomedicine (BBM) at Faculty of Health and Life Sciences, Management and Science University (MSU), Shah Alam, Malaysia. They were chosen randomly and asked to participate. The purpose of this study was told to those who volunteered, and a verbal consent was obtained. Writing the answers was used with participant permission and the interview took approximately 30 minutes. Three students from BMS and one student from BBM were trained by the author to conduct the interview. At the beginning of the meeting, the interviewer introduced himself/herself, briefly explained the study purpose. The interviewers followed the standardized protocol to ensure that all the participants' conducted in a similar manner and that an identical set of questions were discussed. The obtained data were sorted into various categories based on content analysis. Data was analyzed manually.

\section{Results}

A total of 40 students (20 from BBM and 20 from BMS) were interviewed. The age of the students ranged between 18 to 26 years. Thirty two of the participants (80\%) were in the age group 21-23 years. Malay and Indian were the highest participants 17 (42.5\%) each, and followed by Chinese 4 (10\%) (Table 1).

\section{H1N1 and Source of Information}

Regarding the awareness of the H1N1, the majority of the respondents were aware that the swine flu is a disease caused by the H1N1 virus. As far the source of the information regarding to H1N1, the majority of the participants knew about the disease through the mass media such as television, newspaper and the internet. Six of them heard about the disease from their friends. One student said:

I listen to the radio all the time, except in the class. BBM student, 21years old, Indian. 
Table 1: Socio-demographic of the participants.

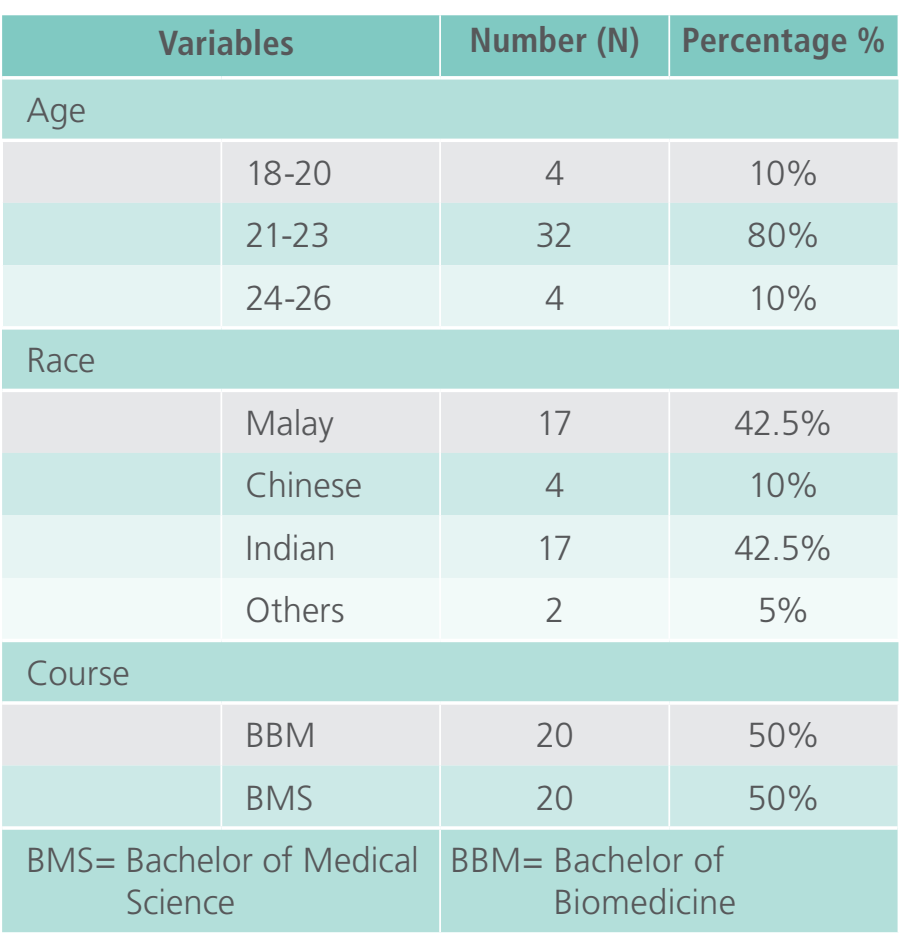

\section{Transmission of the H1N1}

Regarding transmission of the H1N1, more than half of the participants (22) reported that the virus transmitted through air only, five of them mentioned that the transmission moved from pigs to human only, and two of them mentioned that the virus transmitted from human to human only. However, one student stated that the mosquito might transmit the virus to human.

Swine-origin influenza virus can spread through mosquito as a vector.

BBM student, 23 years old, Chinese.

As for the transmission of the H1N1 through the pig's meat, a number of 12 participants thought that the people might be infected by eating pork meat as they believed that the viruses might spread throughout the pig's meat. Twenty one participants thought that eating pork does not cause the H1N1. Five participants were not sure of the answer as they still skeptical whether eating pork might or might not cause the H1N1 infection.
Swine-origin influenza virus cannot be infected by eating pork because the pig's meat does not contain the virus.

BBM student, 22 years old, Indian.

Swine-origin influenza virus cannot be transmitted through eating pig's meat because the virus in the meat is almost dead when cooked.

BBM student, 20 years, Chinese.

\section{The Highest Groups at Risk of H1N1}

Regarding to the highest groups at risk of the H1N1, some of the participants [9] said that pig farmers and those who have close contact with pigs are at high risk of the disease infection, followed by 8 participants who said that travelers are at the highest risk to get the virus infection. Then 7 participants said that anyone exposed to infected people is at the highest risk to get the virus infection. Five participants said that low immune systems of the people are at the highest risk to get the virus infection. Four participants said that health care professionals are at the highest risk to get the virus infection. Four participants said that elderly people and children are at the highest risk to get the virus infection. Two students said that they are not sure who are at the highest risk to get the virus infection. One student answered that pregnant women are at the highest risk infection.

People who travel to infected country and those in contact with pigs are at the highest risk to get the swine-origin virus infection.

BBM student, 22 years old, Indian.

Anyone can get it if they exposed to the infected area.

BBM student, 23 years old, Indian.

Those who visited Mexico and USA recently are at the highest risk to get swine-origin influenza virus.

BBM student, 22 years old, Indian. 
Who is eating pork and stay closed to infected area are at risk to get the swine-origin influenza infection.

BMS student, 22 years old, Malay.

Yes, it is safe to travel to other countries during the swine-origin influenza pandemic; but avoid countries which having infection.

BBM student, 20 years, Chinese.

As far as fatality from the H1N1 virus, the majority of the participants (32) said that the infection might cause fatality. The remaining 8 participants were unsure if the disease might cause fatality or otherwise.

With poor management, the mortality rate is high. BMS student, 23 years old, Malay.

\section{Symptoms of the H1N1 Infection}

Regarding the symptoms of the H1N1 infection, the majority of the participants (34) mentioned that the symptoms of the infection are fever, followed by coughing (12 participants) then vomiting (9 participants), headache (4 participants), and sneezing (4 participants). Few of them reported that fatigue, sore throat, running nose, diarrhea, dizziness, nausea, muscle pain and difficulty of breathing. Rashes, muscle weak, bone pain, back pain, loose appetite, chills are mentioned by one participant. Four participants said that they didn't know any symptoms of the H1N1 infection. One participant answered that the H1N1 is a symptomatic disease. Some participants gave more than one symptom.

Swine-origin influenza is a symptomatic disease. BBM student, 22 years old, Chinese.

Headache, vomiting, difficulty in breathing, cough, sore throat and fever are the main symptoms of the swine-origin influenza.

BBM student, 23 years old, Indian.

\section{Prevention from the H1N1 Virus}

As far as prevention measures of the H1N1 infection, almost half of the participants answered that the infection might be prevented by wearing mask, washing hands and avoiding close contact with infected patients, early prevention and by quarantining the infected patients. Four participants said that by avoiding travelling and avoiding contact with pigs might prevent the spread of the disease. One student said that all infected pigs should be killed and burned as a prevention method.

\section{Kill all infected swine and burn it.}

BBM student, 23years old, Chinese.

By wearing protective masks and frequent hand washing can prevent this infection.

BBM student, 22 years old, Indian.

The best way for prevention now is to wear protection mask and do not travel to affected counties.

BMS student, 23 years old, Malay.

Regarding the vaccination against H1N1 virus, more than half of the participants were quite aware that there is no available vaccine for H1N1 right now. Nine of them said that they were sure there is a vaccine available for H1N1. Four participants did not know whether there are any vaccines available or not.

There is no vaccine available but research is being done for new vaccine.

BBM student, 22 years old Indian.

Yes, there are 4 types of vaccine.

BBM student, 23 years old, Chinese.

No vaccine yet for the swine-origin influenza, only vaccine existed for H5N1.

BMS student, 23 years old, Malay. 


\section{Treatment}

As far as treatment, 16 participants said that the treatment for this disease is by using antiviral drugs. Eleven of them do not know if there are any treatments available for this disease. Six said that the H1N1 infection cannot be treated at all.

The disease might be suppressed using Tamiflu. BBM student, 22 years old, Indian.

To treat the infection by giving antivirus and patient should be quarantined.

BBM student, 23 years old, Indian.

No idea, may there is any treatment.

BMS student, 22 years old, Malay.

\section{The Best Way to Develop Awareness about H1N1 Infection}

As for the best way to develop awareness about this disease, 17 participants thought that the best way is to educate the students of MSU by organizing campaigns and distributing brochures. Eighteen of them reported that it would be a better idea to organize seminars or giving talks explaining about the disease to create better awareness. Five of them suggested that academic lessons and lecturers would do a better job at developing awareness among students.

The best way to create awareness about swineorigin influenza is by organizing campaigns, talks and distributing brochures on the danger of the swine-origin influenza.

BBM student, 22years old, Indian.

Do campaign and explain about this problem and tell them how dangerous this flu.

BMS student, 22 years old, Malay

\section{Discussion}

This study showed that the majority of the respondents were aware that the swine flu is a disease caused by the H1N1 virus. The majority of them knew about the disease through the mass media. Similar findings are reported by Langeroudi and Estabraghi [6] that most responses showed that media was one of the sources of knowledge among students. More than half of the participants reported that the virus transmitted through air. Similar finding was reported that H1N1 transmitted through airborne. [7] The misconception of the transmission reported among the participants is that H1N1 could be transmitted via mosquito bites. Likewise previous study reported that H1N1 could be transmitted through insects. [8] The results of present study showed that 12 out of 40 students thought that the susceptible individual might be infected by eating pork meat as they believed the H1N1 virus spread out through the pig's meat. Regarding to the highest groups at risk of the swine flu, pig farmers and those who have close contact with pigs, travelers, elderly people health care professionals and pregnant women are at high risk of the disease infection [1]. Few of the participants in this study mentioned that the individuals with low immunity are among high risk groups and this was consistent with WHO report. [3] Some other practice with regard to personal hygiene measures for example avoid touching eye and noses had not been mentioned by this study participants, analogous finding was reported by Lau and others. [8] Regarding to the symptoms of the H1N1 infection, the majority of the participants reported that the symptoms of the infection were fever, followed by coughing, vomiting, headache, sneezing fatigue, sore throat and nose running. The results of this study were consistent with recent study by Komiya et al. [9].

As far as prevention measures of the swine flu infection, almost half of the participants answered that the swine flu might be prevented by wearing 
face-masks, washing hands and avoiding close contact with infected patients. Similar finding reported that the participants considered face-masks as an effective measures of protection against H1N1. $[10,8]$ Regarding the vaccination against H1N1, more than half of the participants were well aware that there is no available vaccine. As for treatment, 16 participants said that the treatment for this disease is by using antiviral drugs. This is true since WHO and the Centers for Disease Control and Prevention (CDC) recommended the antiviral agent oseltamivir (OTV) for treatment of infected patients. [11] Some of the students reported that H1N1 cannot be treated. The belief of participants that there is no treatment for H1N1 cases was reported by Lau et al. [8].

\section{Strengths and limitation}

The limitation of this study is the lack of the literature review to compare our study with. The other limitation is that the sample was recruited from single private institute and the results may not be representative of all the university students in Malaysia.

\section{Conclusion}

Despite that the majority of the participants had the basic knowledge regarding H1N1 and its preventions measures such as wearing face-masks, washing hands and avoiding close contact with infected patients, some of them had some misconceptions about the infection, such as the infection might be transmitted by eating pork meat and by mosquito bites. Few students reported that the pig farmers and those in close contact with pigs were at high risk of contracting the disease, followed by travelers.

\section{Recommendations}

Health education for university students regarding personal hygiene in reducing H1N1 transmission is recommended. 


\section{References}

1. WHO, Epidemic and Pandemic Alert and Response (EPR). Available from: http://www.who.int/csr/ don/2009_05_13/en/ index.html>, (accessed May 2009).

2. WHO, Media centre. Influenza A (H1N1). Available from: http://www.who.int/mediacentre/news/ statements/2009/ h1n1_20090429/en/index.html>, (accessed May 2009).

3. WHO, Considerations on influenza A (H1N1) and HIV infection. Available from: http://www.who.int/hiv/mediacentre/influenza_ hiv.pdf, (Accessed 6 August 2009)

4. Disease Control Division Ministry of Health Malaysia. Mitigation Phase: Influenza A (H1n1) Surveillance Strategies. http://h1n1. moh.gov.my/surveillance Strategies/ (17-07-2009).

5. Disease Control Division Ministry of Health Malaysia. Pandemic (H1N1) 2009: Current Situation in Malaysia. http://www.afpm. org.my/v2/articles/Pandemic\%20 (H1N1) (2009).

6. Langeroudi AG and Estabraghi AS. Bird flu knowledge among veterinary medical students in Iran. Journal of Animal and Veterinary Advances 2008; 7:526-528.

7. Brankston G, Leah G, Zahir H, Camille L, \& Michael. Transmission of influenza $A$ in human beings. The Lancet Infectious Diseases 2007; 7: 257-65.

8. Lau JTF, Griffiths S, Choi KC \& Tsui HY. Widespread public misconception in the early phase of the H1N1 influenza epidemic. Journal of Infection 2009; 59:122-127.

9. Komiya N, Gu Y, Kamiya H, Yahata $Y$, et al. Clinical features of cases of influenza $A(H 1 N 1) \vee$ in Osaka prefecture, Japan, Eurosurveillance 2009; 14: 1-3.

10. Cowling BJ, Fung RO, Cheng CK, et al. Preliminary findings of a randomized trial of Non-pharmaceutical interventions to prevent influenza transmission in households. PloS One 2008; 3:E2101

11. Rungrotmongkol $T$, Intharathep $P$, Malaisreeet $M$, et al. Susceptibility of antiviral drugs against 2009 influenza A (H1N1) virus. Biochemical and Biophysical Research Communications 2009; 389:390-394.

\section{Comment on this article:}

\section{(f) in $8+\mathbf{S} P$}

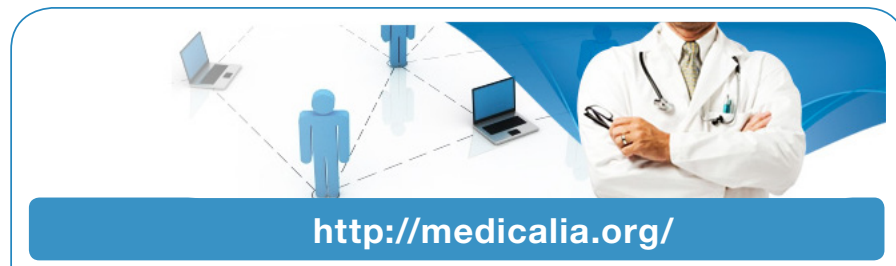

Where Doctors exchange clinical experiences, review their cases and share clinical knowledge. You can also access lots of medical publications for free. Join Now!

\section{Publish with iMedPub}

\section{http://www.imed.pub}

International Archives of Medicine is an open access journal publishing articles encompassing all aspects of medical science and clinical practice. IAM is considered a megajournal with independent sections on all areas of medicine. IAM is a really international journal with authors and board members from all around the world. The journal is widely indexed and classified Q1 in category Medicine. 